\title{
Systems Oncology: Towards patient-specific treatment regimes informed by multiscale mathematical modelling
}

\author{
Gibin G. Powathil ${ }^{\mathrm{a}}$, Mark A.J. Chaplain ${ }^{\mathrm{a}, *}$, Maciej Swat ${ }^{\mathrm{b}}$ \\ ${ }^{a}$ Division of Mathematics, University of Dundee, Dundee, UK, DD1 $4 \mathrm{HN}$. \\ ${ }^{b}$ The Biocomplexity Institute and Department of Physics, Indiana University Bloomington, Bloomington, Indiana, \\ USA.
}

\begin{abstract}
The multiscale complexity of cancer as a disease necessitates a corresponding multiscale modelling approach to produce truly predictive mathematical models capable of improving existing treatment protocols. To capture all the dynamics of solid tumour growth and its progression, mathematical modellers need to couple biological processes occurring at various spatial and temporal scales (from genes to tissues). Because effectiveness of cancer therapy is considerably affected by intracellular and extracellular heterogeneities as well as by the dynamical changes in the tissue microenvironment, any model attempt to optimise existing protocols must consider these factors ultimately leading to improved multimodal treatment regimes. By improving existing and building new mathematical models of cancer, modellers can play important role in preventing the use of potentially sub-optimal treatment combinations. In this paper, we analyse a multiscale computational mathematical model for cancer growth and spread, incorporating the multiple effects of radiation therapy and chemotherapy in the patient survival probability and implement the model using two different cell based modelling techniques. We show that the insights provided by such multiscale modelling approaches can ultimately help in designing optimal patient-specific multi-modality treatment protocols that may increase patients quality of life.
\end{abstract}

Keywords: Hybrid multiscale model, cell-cycle, hypoxia, chemotherapy, radiation therapy

\section{Introduction}

Along with the rapid growth in acquisition of genetic, proteomic and other biochemical and biological data, there has been a parallel development from the theoretical side in terms of modelling. In particular, Systems Biology has emerged as a field of research over the past decade applied to a wide range of problems in the biomedical sciences. Systems Biology seeks to bring to bear a range of inter-disciplinary skills and tools on complex biomedical problems. By adopting a holistic or integrative approach (as opposed to the more traditional reductionist logic), systems biology aims to predict emergent behaviour that will arise from complex biomedical systems i.e. behaviour that appears over time due to the interactions between genes, proteins, cells and tissues across a range of spatial and temporal scales. Given the complexity of most biomedical systems and their inherent nonlinearities, it is not possible to make accurate predictions without adopting some kind of "systems approach". Indeed, in the last few years, systems biology itself has evolved and further developed seeking not just to understand events at the separate biological scales in a qualitative manner, but also to develop mathematical models which are truly multiscale, leading to the emergence of "quantitative systems biology" or "quantitative integrative biology". This novel systems approach is now being brought to bear on cancer modelling and a related discipline of what may be termed Systems Oncology now exists in its own right to develop predictive multiscale models of cancer growth and spread. In this paper we present a brief summary of previous cancer modelling over the past 15-20 years and then focus on two recent multiscale models of a solid tumour undergoing radio- and chemotherapy treatments.

\footnotetext{
${ }^{*}$ Corresponding author:

Email address: chaplain@maths.dundee.ac.uk (Mark A.J. Chaplain)
} 
We show how simulation results can be used to optimize treatment regimes resulting in better clinical outcomes for individual patients.

\section{Mathematical Models in Systems Oncology}

Decades of cancer modeling have produced established models representing all the key phases of solid tumour growth i.e. avascular growth [1,2], tumour-induced angiogenesis [3, 4], the immune response to cancer [5, 6], invasion and metastasis $[7,8,9,10,11]$ and vascular growth $[12,13]$. New areas are also now being investigated concerning the spatio-temporal modelling of intracellular pathways associated with cancer such as p53-Mdmd2 [14, 15]. A comprehensive overview of the field may be found in the review article of Lowengrub et al. [16]. In the past few years especially, multiscale models of solid tumour growth have been developed in order to account for the different spatial and temporal scales (from genes to tissues) that occur not only in cancer but in all biological systems $[17,18,19,20]$. A review of recent models in this area may be found in the paper of Deisboeck et al. [21]. There has also been a concerted effort to integrate mathematical models of cancer with real data in an attempt to develop quantitative, predictive models of cancer progression $[22,23]$ and its treatment using chemotherapy [24, 25, 26, 27, 28, 29, 30], radiotherapy [31, 32, 33].

Most of the recent multiscale models of cancer growth and treatment adopt some kind of individualbased modelling approach where the individual cell is the initial focus of the model. Intracellular processes may be incorporated through systems of ordinary differential equations modelling processes in the cytoplasm and nucleus being associated with each individual cell. By simulating many interacting cells, insight into emergent tissue level phenomena can be achieved. In the next section we briefly review some of the cell-based modelling techniques currently being adopted before giving two specific examples of multiscale models of a solid tumour undergoing radio- and chemotherapy treatments.

\section{Cell-based Modelling Techniques}

Cancer is a complex disease involving many interrelated processes that occur across a wide range of spatial and temporal scales, from the intra- cellular level to the tissue level. Consequently, multiscale models are needed to capture these coupled processes. In the past 5 years or so, researchers have developed several mathematical and computational techniques that allow the study of how singlecell-based behaviours and local cell-cell and cell-ECM interactions lead to emergent phenomena at the tissue level. These methods "trade off" the level of detail per cell against the number of cells per simulation. A key realisation that lies at the foundation of all these methods is that tissue development, homeostasis or disease are all driven by a fairly limited set of cellular behaviours. By growing, dividing, dying, adhering, secreting and absorbing chemicals or transducing signals and interacting with extracellular matrix, cells give rise to a whole range of tissue-level phenomena from healthy tissue development to cancer metastasis. Since cells live in a highly viscous environment all cell-based modelling methods assume some kind of relation between force $(\mathbf{F})$ and cell velocity $(\mathbf{v})$.

The simplest technique to implement (but not always the simplest to analyse) is the cellular automaton (CA) model which represent cells as single lattice points and encodes cellular behaviours in terms of transition probabilities of cells moving from one lattice location to another. Although defining rules and probabilities governing transitions in CA model is easy, linking these rules to forces or other physically measurable cellular characteristics is quite challenging. However simplifying single cell representations allows modellers to simulate large tissue fragments within reasonable computation times [34, 35, 36]. Another related approach is based on lattice-gas cellular automaton approach [37]. Center Models (CM) $[38,39]$ relax the requirement of cells to be constrained to the grid points on a fixed lattice by representing cells as interacting points in space. Inspired by molecular dynamics, CMs solve force-velocity, $\mathrm{F} \sim \mathrm{v}$, equations for each of the model cells. CMs force- or energy-based formalism permits a much easier translation of measurable quantities into model parameters. Although CMs are computationally more challenging than Cellular Automaton models they provide more biophysical detail and also enable simulation up to the level of large tissue fragments. Sub-cellular Element Models (SEM) appear to be a natural extension of CMs and they represent an individual cell as a 
collection of interacting points in space. To faithfully represent a single cell morphology and to keep model cells unfragmented, SEM models apply "strong" interactions between points belonging to same cell and somewhat weaker interactions to points that are members of different cells. This separation of interaction strengths makes numerical integration of equations of motions more challenging [40] as compared to the simpler Center Model implementation. Regardless of this, numerical treatment of all center models (including SEM) is much simpler than molecular dynamics where one cannot assume a F $\sim$ v relation. SEMs permit the detailed simulation of single cell morphology, shape changes, etc. but as anticipated, the average size of the simulation in terms of number of cells is several orders of magnitudes smaller than in the case of CA and CMs. The Cellular Potts Model (CPM) (or Glazier-Graner-Hogeweg (GGH) model) [41] is a stochastic method that approximates complex cell shapes as collections of pixels on a regular lattice and defines their behaviours and interactions through the local minimization of effective energies depending on cell and pixel configurations. By minimizing energy via the Modified Metropolis Algorithm, CPM recovers a linear relation between force and a cells velocity and by using an energy-based formalism it allows the translation of labmeasurable cellular characteristics into model parameters. The CPM embeds the $\mathrm{F} \sim \mathrm{v}$ relation into a stochastic computational algorithm making it less explicit than in center models. Using a lattice makes CPM simulations faster than corresponding SEM simulations but not as fast as CA or simple center models. When details of single-cell representations are important, the Finite Element (FE) technique and Immersed Boundary (IB) method [42] provide viable alternatives to earlier methods but at much greater computation cost per cell. Ultimately, each simulation method should give the same results for the same biologically-determined classes of objects, behaviours and interactions. Any observed discrepancies between methods can used to veto and/or improve modelling methods.

In the remainder of this paper we focus on two recent multiscale models of a solid tumour undergoing radio- and chemotherapy treatments. We show how the computational simulation results can be used to optimize treatment regimes resulting in better clinical outcomes for individual patients.

\section{A Multiscale Mathematical Model of Multimodality Treatment}

The growth and progression of a solid tumour mass depends critically on the responses of the individual cells that constitute the entire tumour mass. The evolution of each individual cancer cell and its decisions to grow, divide, remain inactive or die are usually influenced by cells spatial location within the tumour and intracellular interactions (e.g. the intracellular cell-cycle). These cellular responses are actively influenced by various extracellular signals from neighbouring cells as well as its dynamically changing microenvironment. Here, we discuss a multiscale mathematical model (and its two different mathematical implementations) for solid tumour progression incorporating such intracellular, cellular and microenvironmental factors. This model can be used to study the effects of cancer treatments and to optimize treatment regimes. The cell-cycle plays a critical role in most of the complex cellular processes that are involved in cancer progression (proliferation, cell division and DNA replication). Within a mammalian cell cel-cycle is controlled by a family of cyclin dependent kinases $(\mathrm{Cdk})$, whose activity is primarily dependent on association with a regulatory protein called cyclin $[43,44]$. Factors such as Cdk inhibitors can act as negative regulators of the cell-cycle and tumour microenvironment [45]. In our cell-based model the growth and proliferation of each cancer cell is determined by its own internal cell-cycle which we model using a set of ordinary differential equations representing the chemical interactions involved in the cell-cycle - see Figure 1 and [36] for details.

The cell-cycle dynamics are critically influenced by its external surrounding tumour microenvironment and in particular, the availability of oxygen and nutrients for the rapidly increasing tumour cell population [43]. Although, tumour cells can proliferate in the absence of oxygen (via glycolysis), the availability of oxygen stimulates rapid proliferation and maintains the function of cell-cycle within the cell. Experiments show that low supply of oxygen (hypoxia) upregulates some of the cell-cycle inhibitory proteins such as p27 and p21 which could impair the cell-cycle, eventually taking the cell either to a resting phase or inducing a cell-cycle arrest [46].

In our 2D model we assume that oxygen is supplied by randomly distributed blood vessels (red cells in the concentration plot shown in Figure 1) [36] that are are perpendicular to the tissue cross 
section with no branching through the plane of interest. In a 3D domain, this can be replaced by a network of vessels with vascular dynamics which are ignored for simplicity in this model. The spatio-temporal evolution of the oxygen concentration $(K(x, t))$ is modelled using a reaction diffusion equation as given in the Figure 1. Here, $m(x)$ denotes the vessel cross section at position $x(m(x)=1$ for the presence of blood vessel at position $x$, and zero otherwise) and $r(x)$ describes rate of oxygen supply [36]. This equation is solved using no-flux boundary conditions and an initial condition of $100 \%$ oxygen distribution [47]. As the tumour cells grow, some them (within the tumour mass) will be starved of oxygen and eventually become hypoxic. These hypoxic conditions further upregulate various proteins and intracellular pathways involved in intracellular growth and metastatic cascade, and cell-cycle dynamics. These changes in the tumour microenvironment are mostly due to the transcription of various physiologically important genes which are activated through the hypoxia inducible transcription factor (HIF -1) pathway [46, 48, 49]. Usually, the tissue is considered hypoxic if the average oxygen concentration falls below $10 \mathrm{~mm} \mathrm{Hg}$ [48] and in our model, we assume that HIF- $1 \alpha$ at position $x$, which is normally inactive, is activated if the oxygen concentration, $K(x, t)$, falls below $10 \%$. To model hypoxia induced cell-cycle inhibitory effects we use an addition term in equation governing Cyclin-CDK dynamics (Equation 1 of the cell-cycle dynamics in Figure 1).

\subsection{Anticancer treatments}

Chemotherapy and radiotherapy play important roles in the primary treatment of many cancers and in improving the survival after surgery. Their effectiveness is affected by intra-tumoural heterogeneity and the changes in its surrounding microenvironment [50]. Consequently, consideration of these dynamical changes could inform the design of optimal therapeutic protocols that improve the therapeutic outcomes.Using our model, we analyse the effects of cell-cycle phase specific chemotherapeutic drugs alone and in combination with radiation therapy in controlling the tumour progression. Because cell-cycle stage-specific chemotherapy targets proteins (e.g.CDKs) that are over expressed during various stages of cancer progression it can control cell growth. Moreover, an appropriate combination of cell-cycle phase targeting drugs can address the occurrence of cell-cycle mediated drug resistance due to the presence of functionally heterogenous cells [51]. We assume that the concentration of chemotherapeutic drug (delivered through vascular cross sections and undergoing natural decay/clearance) also follow a similar dynamics as that of oxygen distribution and is governed by a similar reaction-diffusion equation as shown in Figure 1. The direct cellular effects after the radiation therapy is modelled using a modified linear quadratic (LQ) model (Figure 1) by considering multiple radiobiological effects due to the irradiation [52]. Previous experiments indicate that the relative radiation sensitivity of a cancer cell depends on multiple factors, including its oxygenation status and its cell-cycle phase $[53,54,55]$. While the cells are found to be more sensitive when in the S-G2-M phase as compared with the G1 phase, their relative radiation sensitivity is minimal at low oxygen levels. We model the effects of cell-cycle phase dependent radio-sensitivity stochastically using parameter $\gamma$ that varies from 0 to 1 , depending on the cell-cycle phases and thus increasing or decreasing the survival chances of an irradiated cell. The effect of changing tissue oxygen levels within the spatial domain on the radiation sensitivity is included in the modified LQ model by using the oxygen modification factor (OMF) [47] and is shown in Figure 1. This OMF introduces a higher probability of cell survival in the absence of oxygen and increases the cell-kill if the cell is well oxygenated. Our models also account for the chances of cellular repair after the radiation and the cell-cycle delay in repairing the cellular damage as it might further affect the cellular redistribution and efficacy of additional doses of multi-modality treatments.

\subsection{Computational methods}

To implement our model we used two different multiscale modelling techniques - Cellular Automaton (CA) and Cellular Potts Model (CPM) $[36,52,56]$. The CA model was implemented using custom $\mathrm{C}++$ /Matlab programs and the CPM-based model was implemented using CompuCell3D [57]. Both implementations use three different components: (1) cancer cells whose spatio-temporal evolution is controlled by internal cell-cycle dynamics; (2) the oxygen/chemotherapeutic drug diffusive fields; (3) 
cross-sections of blood vessels delivering oxygen and chemotherapeutic drugs - see Figure 2 for details. We simulated the CA model (corresponding to $2 \times 2 \mathrm{~mm}^{2}$ of cancer tissue) on a $100 \times 1002 \mathrm{D}$ grid with lattice spacing set to $l=20 \mu \mathrm{m}$ thus allowing each lattice site to accommodate one cancer cell (or remain empty). For a CPM-based simulation, we have used a $300 \times 300$ pixels 2D lattice. CPM [7] represents each biological cell as spatially extended domains that is collection of lattice pixels having the same index marker. In both model implementations, the simulation begins with a single tumour cell whose proliferation is governed by the internal cell-cycle dynamics. The intracellular dynamics are solved in CA model using Runga-Kutta method. For CPM-based simulation we use CompuCell3D SBML Solver modules to solve ODEs [56]. The oxygen and drug dynamics are solved using a finite difference methods. The cell-cycle phases of each cancer cell is determined using the concentration levels of $[\mathrm{CycB}]$. If $[\mathrm{CycB}]$ is greater than a specific threshold (we use 0.1) the cell is considered to be in the S-G2-M phase (green cell) and if it is lower than this value, the cell is in the G1-phase. If the cyclinB-cdk complex concentration $[\mathrm{CycB}]$ crosses this threshold from above, the cell undergoes cell division and its mass [mass] or [volume] is halved. Further details of both the simulation approaches that are used to study the multiscale mathematical model and the relevant parameter values can be found in recent papers by Powathil et al. [36, 52, 56].

\subsection{Computational Simulation Results}

The main goals of cancer modelling is to develop simulations that can help designing new and optimize existing treatment modes that improve clinical outcomes. Presented model developed by Powathil et al. [36] allows to study the responses of individual cancer cells to the given doses of cell-cycle phase specific chemotherapy, radiotherapy and their combinations.

Figure 3 shows the spatial evolution of the tumour cells, indicating their respective cell-cycle phase and oxygenation status, simulated using CA (Figure 3.i) and CPM approach (Figure 3.ii). Although each of these modelling approach is technically different, the results obtained from both multiscale models are similar and comparable, showing the robustness of the multiscale mathematical modelling approach. While it does not confirm the accuracy of the model itself it does provide an important cross-validation case-study between different computational implementations of the same biological model. It also increases our confidence that presented model can in fact suggest better treatment regimes.

Cell-cycle phase-specific chemotherapeutic drugs used in treating various cancer malignancies interfere with the rapidly proliferating mass of the cells by blocking their cell division cycle in respective cell-cycle phases. Cell-cycle phase specific chemotherapeutic drugs can interact with the divisional cycle in multiple ways - some target cells in $\mathrm{S}$ phase, interfering with its replication, resulting a celldeath or cell-cycle arrest at divisional checkpoints while others damage the formation of the mitotic spindle or prevent it from disassociating, targeting the cells in $M$ phase. There are also drugs that can block transition from one phase to another at several transitional checkpoints, preventing the cell division. Although, the concept of phase-specificity is sometimes an over simplification as most of the drugs affects more than one phases, in our model we consider the effects of two types of phase-specific chemotherapeutic drugs that are either G1 specific or G2-S-M specific on a growing tumour.

We simulate two doses of either types of the drugs that delivered at a same rate at times 500 hours and 550 hours. A representative figure, Figure 4 shows the spatio-temporal evolution of cancer cells when the tumour mass is treated with two doses of G1 phase-specific drugs and G2 phase-specific drugs. The plots in the Figure 4 show that a combination of G1 specific drug followed by another G1 specific drug give better therapeutic outcome than two G2 drug combinations. This is due to the presence of a higher fraction of proliferating cells in G1-phase at the time of the drug doses and increased proliferation after the initial dose. Interestingly, these drug combinations need not always give the best outcome, especially if there were a higher proportion of resting cells within a growing tumour mass [36]. An earlier detailed analysis of the described multiscale mathematical model [36, 56] indicated that in addition to the phase-specificity of the chemotherapeutic drug, their sequencing and scheduling significantly affect the spatial distribution and effectiveness of the chemotherapy. Moreover, the changes in the spatial distribution of tumour cell mass due to the cytotoxic cell-kill further affect the surrounding microenvironment and intracellular cell-cycle dynamics and thus have impact on 
cytotoxic effects of subsequent doses of the chemotherapeutic drug. Hence, the knowledge of spatial distribution of a growing tumour mass and the intra- and extra-cellular heterogeneities is needed to achieve better cytotoxic effectiveness and the best possible treatment outcomes.

Similarly, radiosensitivity of an irradiated cells and its radiobiological responses are very much affected by various intracellular and extracellular factors and in particular, cell-cycle dynamics and availability of tissue oxygen [54]. In addition, the delivered radiation fractions further dynamically change this radiosensitivity over time by redistributing the cancer cells within the cell-cycle, by inducing repopulation of the tumour mass, by allowing reoxygenation of the tumour, and by activating DNA repair mechanisms (5 R's of radiation) $[58,54]$. As the traditional linear quadratic model was too simple to account for these multiple factors, we have introduced a cell-based modified LQ model to study the cellular responses after the radiation [52]. We calculated the survival probability of each irradiated cell based on this modified LQ model and used this model to to study various factors affecting the radiation sensitivity such as hypoxia, intracellular repair mechanism and radiation fractions $[52]$.

The accuracy of the developed model is analysed by comparing the simulation results of the model with the experimental results when the cells are irradiated with a radiation dose of 3 Gy [59, 52]. The results of the comparison are given in the Figure 5. Figure 5.i shows the experimental results that give the percentage of cells in G1 and G2 phases with and without irradiation and Figure 5.ii gives the corresponding simulation results. The experimental results show that the cells in the control case are predominantly distributed in G1 phase than G2 phase. After irradiation, the majority of the cells started to accumulate in G2 phase, about $12 \mathrm{~h}$ after irradiation and stayed in G2 phase before going back to a G1 phase dominant cell distribution by $22-24 \mathrm{~h}$ after irradiation [59]. The results obtained from the multiscale mathematical model are qualitatively in a good agreement with these experimental findings and Figures 4 and 5 give good description how tumour morphology looks like after single dose of radio- and/or chemo-therapy. By modelling subsequent doses of cytotoxic factors we can analyze treatment process in more detail and use this knowledge as an initial guess in optimizing treatment doses/scheduling. Moreover, the analysis of the radiobiological responses after the irradiation further indicated that the radiation can induce full/partial cell-cycle synchronisation during the therapeutic regime, showing its usefulness in studying and planning kinetic administration of multi-modality treatment protocols.

\section{Conclusions and Future Perspectives}

Many recent multiscale models of cancer growth and treatment have adopted some kind of individualbased modelling approach where interacting individual cells are the initial focus of the model. By incorporating intracellular processes for each cell (through systems of ODEs) and then simulating many interacting cells, insight into emergent tissue level phenomena can be achieved. In this paper we have focussed on two recent multiscale models of a solid tumour undergoing radio- and chemotherapy treatments. We demonstrated how the computational simulation results can be used to optimize treatment regimes.

The insights provided by the multiscale models showed the importance of considering multiple intracellular and extracellular factors that are critical in accessing cancer progression and treatment response to devise an optimal multi-modality treatment regime. As these intracellular and extracellular processes occur at different spatial and temporal scales, development of multiscale mathematical models and their implementation through multiscale computational techniques is the next step forward in interdisciplinary systems medicine and systems oncology modelling. This reasoning applies not only to models of the growth and progression of and cancer (angiogenesis, invasion, metastasis), but also to the various treatment protocols currently in use (radiotherapy, chemotherapy and combinations thereof).

Furthermore, when considering treatment regimes, in addition to the direct cytotoxic effect to the growing tumour mass, the anticancer therapies induce indirect effects on surrounding tumour cells, normal cells and its microenvironment that might further affect the cumulative effectives of the multimodality treatments. For example, when the tumour cells are treated with radiation therapy, 
the surviving tumour cells and its surrounding normal cells are further affected by so called "radiation induced bystander effects". The term "radiation-induced bystander effect" is used to describe radiation-induced biological changes that occur in cells that have not been directly irradiated [60]. In the case of chemotherapy, the cytotoxic effectiveness is negatively affected by heterogeneities caused by multiple mutated subpopulations with varying drug sensitivities and other intrinsic and acquired multi drug resistance. In future modelling studies, we will incorporate these additional mechanisms that indirectly affect the effectiveness of chemotherapy and radiation therapy.

The long-term goal of all such modelling is the development of quantitative, predictive models based on clinical and experimental data which will no doubt have a positive impact on patients suffering from diseases such as cancer through improved clinical treatment i.e. the development of patient-specific treatment regimes informed and optimised by multiscale mathematical modelling.

\section{Acknowledgments}

The authors gratefully acknowledge the support of the ERC Advanced Investigator Grant 227619, M5CGS: From Mutations to Metastases: Multiscale Mathematical Modelling of Cancer Growth and Spread.

\section{References}

[1] Byrne HM, Chaplain MA. Growth of nonnecrotic tumors in the presence and absence of inhibitors. Math Biosci. 1995 Dec;130(2):151-181.

[2] Byrne HM, Chaplain MA. Growth of necrotic tumors in the presence and absence of inhibitors. Math Biosci. 1996 Jul;135(2):187-216.

[3] Anderson AR, Chaplain MA. Continuous and discrete mathematical models of tumor-induced angiogenesis. Bull Math Biol. 1998 Sep;60:857-899.

[4] McDougall SR, Anderson AR, Chaplain MA. Mathematical modelling of dynamic adaptive tumour-induced angiogenesis: clinical implications and therapeutic targeting strategies. J Theor Biol. 2006 Aug;241(3):564-589.

[5] Al-Tameemi M, Chaplain M, d'Onofrio A. Evasion of tumours from the control of the immune system: consequences of brief encounters. Biol Direct. 2012;7:31.

[6] Matzavinos A, Chaplain MA, Kuznetsov VA. Mathematical modelling of the spatio-temporal response of cytotoxic T-lymphocytes to a solid tumour. Math Med Biol. 2004 Mar;21(1):1-34.

[7] Andasari V, Gerisch A, Lolas G, South AP, Chaplain MA. Mathematical modeling of cancer cell invasion of tissue: biological insight from mathematical analysis and computational simulation. J Math Biol. 2011 Jul;63(1):141-171.

[8] Deakin NE, Chaplain MA. Mathematical modeling of cancer invasion: the role of membranebound matrix metalloproteinases. Front Oncol. 2013;3:70.

[9] Gerisch A, Chaplain MA. Mathematical modelling of cancer cell invasion of tissue: local and non-local models and the effect of adhesion. J Theor Biol. 2008 Feb;250(4):684-704.

[10] Ramis-Conde I, Chaplain MA, Anderson AR, Drasdo D. Multi-scale modelling of cancer cell intravasation: the role of cadherins in metastasis. Phys Biol. 2009;6(1):016008.

[11] Ramis-Conde I, Drasdo D, Anderson AR, Chaplain MA. Modeling the influence of the Ecadherin-beta-catenin pathway in cancer cell invasion: a multiscale approach. Biophys J. 2008 Jul;95(1):155-165. 
[12] Macklin P, McDougall S, Anderson AR, Chaplain MA, Cristini V, Lowengrub J. Multiscale modelling and nonlinear simulation of vascular tumour growth. J Math Biol. 2009 Apr;58(45):765-798.

[13] Wu M, Frieboes HB, McDougall SR, Chaplain MA, Cristini V, Lowengrub J. The effect of interstitial pressure on tumor growth: coupling with the blood and lymphatic vascular systems. J Theor Biol. 2013 Mar;320:131-151.

[14] Sturrock M, Terry AJ, Xirodimas DP, Thompson AM, Chaplain MA. Spatio-temporal modelling of the Hes1 and p53-Mdm2 intracellular signalling pathways. J Theor Biol. 2011 Mar;273(1):1531.

[15] Sturrock M, Terry AJ, Xirodimas DP, Thompson AM, Chaplain MA. Influence of the nuclear membrane, active transport, and cell shape on the Hes1 and p53-Mdm2 pathways: insights from spatio-temporal modelling. Bull Math Biol. 2012 Jul;74(7):1531-1579.

[16] Lowengrub JS, Frieboes HB, Jin F, Chuang YL, Li X, Macklin P, et al. Nonlinear modelling of cancer: bridging the gap between cells and tumours. Nonlinearity. 2010;23:R1-R9.

[17] Alarcon T, Byrne HM, Maini PK. A cellular automaton model for tumour growth in inhomogeneous environment. J Theor Biol. 2003 Nov;225:257-274.

[18] Alarcon T, Byrne HM, Maini PK. A multiple scale model for tumour growth. Multiscale Model Sim. 2005;3:440-475.

[19] Ribba B, Saut O, Colin T, Bresch D, Grenier E, Boissel JP. A multiscale mathematical model of avascular tumor growth to investigate the therapeutic benefit of anti-invasive agents. J Theor Biol. 2006 Dec;243(4):532-541.

[20] Zhang L, Wang Z, Sagotsky JA, Deisboeck TS. Multiscale agent-based cancer modeling. J Math Biol. 2009 Apr;58(4-5):545-559.

[21] Deisboeck TS, Wang Z, Macklin P, Cristini V. Multiscale cancer modeling. Annu Rev Biomed Eng. 2011 Aug;13:127-155.

[22] Byrne HM. Dissecting cancer through mathematics: from the cell to the animal model. Nat Rev Cancer. 2010 Mar;10:221-230.

[23] Billy F, Ribba B, Saut O, Morre-Trouilhet H, Colin T, Bresch D, et al. A pharmacologically based multiscale mathematical model of angiogenesis and its use in investigating the efficacy of a new cancer treatment strategy. J Theor Biol. 2009 Oct;260(4):545-562.

[24] Agur Z, Hassin R, Levy S. Optimizing chemotherapy scheduling using local search heuristics. Operations Research. 2006 Sep-Oct;54(5):829-846.

[25] Fister KR, Panetta JC. Optimal control applied to cell-cycle-specific cancer chemotherapy. SIAM Journal on Applied Mathematics. 2000;p. 1059-1072.

[26] Frieboes HB, Edgerton ME, Fruehauf JP, Rose FR, Worrall LK, Gatenby RA, et al. Prediction of drug response in breast cancer using integrative experimental/computational modeling. Cancer Res. 2009 May;69:4484-4492.

[27] Liu W, Hillen T, Freedman H. A mathematical model for M-phase specific chemotherapy including the G0-phase and immunoresponse. Mathematical Biosciences and Engineering. $2007 ; 4(2): 239$.

[28] Mistry HB, MacCallum DE, Jackson RC, Chaplain MA, Davidson FA. Modeling the temporal evolution of the spindle assembly checkpoint and role of Aurora B kinase. Proc Natl Acad Sci USA. 2008 Dec;105(51):20215-20220. 
[29] Panetta J, Adam J. A mathematical model of cycle-specific chemotherapy. Mathematical and computer modelling. 1995;22(2):67-82.

[30] Ribba B, Marron K, Agur Z, Alarcon T, Maini PK. A mathematical model of Doxorubicin treatment efficacy for non-Hodgkin's lymphoma: investigation of the current protocol through theoretical modelling results. Bull Math Biol. 2005 Jan;67(1):79-99.

[31] Enderling H, Anderson AR, Chaplain MA, Munro AJ, Vaidya JS. Mathematical modelling of radiotherapy strategies for early breast cancer. J Theor Biol. 2006 Jul;241(1):158-171.

[32] Ribba B, Colin T, Schnell S. A multiscale mathematical model of cancer, and its use in analyzing irradiation therapies. Theor Biol Med Model. 2006;3:7.

[33] Richard M, Kirkby K, Webb R, Kirkby N. A mathematical model of response of cells to radiation. Nuclear Instruments and Methods in Physics Research Section B: Beam Interactions with Materials and Atoms. 2007;255(1):18-22.

[34] Moreira J, Deutsch A. Cellular Automaton Models Of Tumor Development: A Critical Review. Advances in Complex Systems (ACS). 2002;5(02):247-267.

[35] Gerlee P, Anderson AR. An evolutionary hybrid cellular automaton model of solid tumour growth. J Theor Biol. 2007 Jun;246:583-603.

[36] Powathil GG, Gordon KE, Hill LA, Chaplain MA. Modelling the effects of cell-cycle heterogeneity on the response of a solid tumour to chemotherapy: Biological insights from a hybrid multiscale cellular automaton model. J Theor Biol. 2012 May;308:1-9.

[37] Dormann S, Deutsch A. Modeling of self-organized avascular tumor growth with a hybrid cellular automaton. In silico biology. 2002;2(3):393-406.

[38] Drasdo D. Center-based single-cell models: An approach to multi-cellular organization based on a conceptual analogy to colloidal particles. In: Single-Cell-Based Models in Biology and Medicine. Springer; 2007. p. 171-196.

[39] Macklin P, Edgerton ME, Thompson AM, Cristini V. Patient-calibrated agent-based modelling of ductal carcinoma in situ (DCIS): from microscopic measurements to macroscopic predictions of clinical progression. J Theor Biol. 2012 May;301:122-140.

[40] Newman TJ. Modeling multicellular structures using the subcellular element model. In: SingleCell-Based Models in Biology and Medicine. Springer; 2007. p. 221-239.

[41] Glazier JA, Balter A, Popławski NJ. Magnetization to morphogenesis: a brief history of the Glazier-Graner-Hogeweg model. In: Single-Cell-Based Models in Biology and Medicine. Springer; 2007. p. 79-106.

[42] Rejniak KA. An immersed boundary framework for modelling the growth of individual cells: an application to the early tumour development. J Theor Biol. 2007 Jul;247(1):186-204.

[43] Douglas RM, Haddad GG. Genetic models in applied physiology: invited review: effect of oxygen deprivation on cell cycle activity: a profile of delay and arrest. J Appl Physiol. 2003 May;94:2068 2083.

[44] Schwartz GK, Shah MA. Targeting the cell cycle: a new approach to cancer therapy. J Clin Oncol. 2005 Dec;23:9408-9421.

[45] Garrett MD, Fattaey A. CDK inhibition and cancer therapy. Curr Opin Genet Dev. 1999 Feb;9:104-111.

[46] Goda N, Ryan HE, Khadivi B, McNulty W, Rickert RC, Johnson RS. Hypoxia-inducible factor 1alpha is essential for cell cycle arrest during hypoxia. Mol Cell Biol. 2003 Jan;23:359-369. 
[47] Powathil G, Kohandel M, Milosevic M, Sivaloganathan S. Modeling the spatial distribution of chronic tumor hypoxia: implications for experimental and clinical studies. Comput Math Methods Med. 2012;2012:410602.

[48] Vaupel P. Oxygenation of human tumors. Strahlenther Onkol. 1990 Jun;166:377-386.

[49] Pouyssegur J, Dayan F, Mazure NM. Hypoxia signalling in cancer and approaches to enforce tumour regression. Nature. 2006 May;441:437-443.

[50] Bailar JC, Gornik HL. Cancer undefeated. N Engl J Med. 1997 May;336:1569-1574.

[51] Shah MA, Schwartz GK. Cell cycle-mediated drug resistance: an emerging concept in cancer therapy. Clin Cancer Res. 2001 Aug;7:2168-2181.

[52] Powathil GG, Adamson DJA, Chaplain MAJ. Towards predicting the response of a solid tumour to chemotherapy and radiotherapy treatments: Clinical insights from a computational model. PLOS Computational Biology (To appear). 2013 June;.

[53] Alper T, Howard-Flanders P. Role of oxygen in modifying the radiosensitivity of E. coli B. Nature. 1956 Nov;178(4540):978-979.

[54] Pawlik TM, Keyomarsi K. Role of cell cycle in mediating sensitivity to radiotherapy. Int J Radiat Oncol Biol Phys. 2004 Jul;59(4):928-942.

[55] Wouters BG, Brown JM. Cells at intermediate oxygen levels can be more important than the "hypoxic fraction" in determining tumor response to fractionated radiotherapy. Radiat Res. 1997 May;147(5):541-550.

[56] Powathil GG, Chaplain MAJ. Multiscale computational modelling of cell-cycle-mediated drug resistance and its implications in cell-cycle-dependent chemotherapy. IET Systems Biology (submitted);

[57] Swat MH, Thomas GL, Belmonte JM, Shirinifard A, Hmeljak D, Glazier JA. Multi-scale modeling of tissues using CompuCell3D. Methods Cell Biol. 2012;110:325-366.

[58] Maity A, McKenna WG, Muschel RJ. The molecular basis for cell cycle delays following ionizing radiation: a review. Radiother Oncol. 1994 Apr;31(1):1-13.

[59] Chaudhry MA. Base excision repair of ionizing radiation-induced DNA damage in G1 and G2 cell cycle phases. Cancer Cell Int. 2007;7:15.

[60] Munro AJ. Bystander effects and their implications for clinical radiotherapy. J Radiol Prot. 2009 Jun;29(2A):A133-142. 


\section{Figures}

Cell-cycle dynamics

$\frac{d[\mathrm{CycB}]}{d t}=k_{1}-\left(k_{2}^{\prime}+k_{2}^{\prime \prime}[\mathrm{Cdh} 1]+[\mathrm{p} 27 / \mathrm{p} 21][H I F]\right)[\mathrm{CycB}]$,

$\frac{d[\mathrm{Cdh} 1]}{d t}=\frac{\left(k_{3}^{\prime}+k_{3}^{\prime \prime}\left[\mathrm{p} 55 \mathrm{cdc}_{\mathrm{A}}\right]\right)(1-[\mathrm{Cdh} 1])}{J_{3}+1-[\mathrm{Cdh} 1]}-\frac{k_{4}[\mathrm{mass}][\mathrm{CycB}][\mathrm{Cdh} 1]}{J_{4}+[\mathrm{Cdh} 1]}$,

$\frac{d\left[\mathrm{p} 55 \mathrm{cdc}_{\mathrm{T}}\right]}{d t}=k_{5}^{\prime}+k_{5}^{\prime \prime} \frac{([\mathrm{CycB}][\text { mass }])^{n}}{J_{5}^{n}+([\mathrm{CycB}][\text { mass }])^{n}}-k_{6}\left[\mathrm{p} 55 \mathrm{cdc}_{\mathrm{T}}\right]$,

$\frac{d\left[\mathrm{p} 55 \mathrm{cdc}_{\mathrm{A}}\right]}{d t}=\frac{k_{7}[\mathrm{Plk} 1]\left(\left[\mathrm{p} 55 \mathrm{cdc}_{\mathrm{T}}\right]-\left[\mathrm{p} 55 \mathrm{cdc}_{\mathrm{A}}\right]\right)}{J_{7}+\left[\mathrm{p} 55 \mathrm{cdc}_{\mathrm{T}}\right]-\left[\mathrm{p} 55 \mathrm{cdc}_{\mathrm{A}}\right]}-\frac{k_{8}[\mathrm{Mad}]\left[\mathrm{p} 55 \mathrm{cdc}_{\mathrm{A}}\right]}{J_{8}+\left[\mathrm{p} 55 \mathrm{cdc}_{\mathrm{A}}\right]}-k_{6}\left[\mathrm{p} 55 \mathrm{cdc}_{\mathrm{A}}\right]$,

$\frac{d[\mathrm{Plk} 1]}{d t}=k_{9}[\operatorname{mass}][\mathrm{CycB}](1-[\mathrm{Plk} 1])-k_{10}[\mathrm{Plk} 1]$,

$\frac{d[\text { mass }]}{d t}=\mu[$ mass $]\left(1-\frac{[\text { mass }]}{m_{*}}\right)$,

Oxygen dynamics

$\frac{\partial K(x, t)}{\partial t}=\nabla \cdot\left(D_{K}(x) \nabla K(x, t)\right)+r(x) m(x)-\phi K(x, t) \operatorname{cell}(x, t)$

Drug dynamics

$\frac{\partial C_{i}(x, t)}{\partial t}=\nabla \cdot\left(D_{c i}(x) \nabla C_{i}(x, t)\right)+r_{c i}(x) m(x)-\phi_{c i} C_{i}(x, t) \operatorname{cell}(x, t)-\eta_{c i} C_{i}(x, t)$

Radiation effects

$S(d)=\exp \left[\gamma\left(-\alpha . \mathrm{OMF} . d-\beta(\mathrm{OMF} . d)^{2}\right)\right]$

where

$$
\mathrm{OMF}=\frac{\operatorname{OER}\left(p O_{2}\right)}{\operatorname{OER}_{m}}=\frac{1}{\operatorname{OER}_{m}} \frac{\operatorname{OER}_{m} \cdot p O_{2}(x)+K_{m}}{p O_{2}(x)+K_{m}}
$$

Hybrid multiscale modelling techniques

Hybrid cellular potts model (Compucell3D)
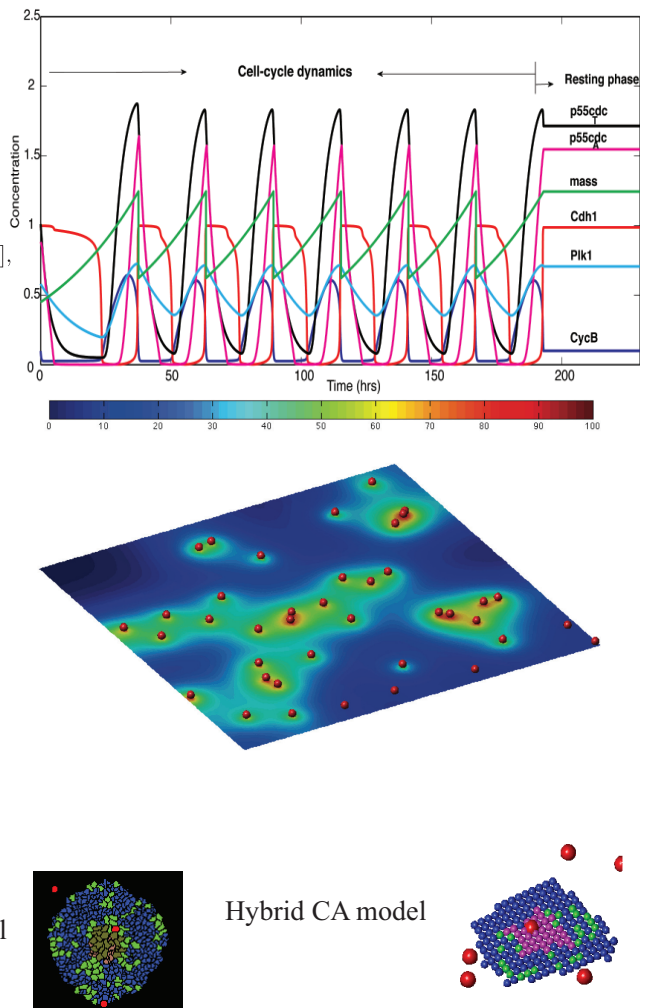

Hybrid CA model

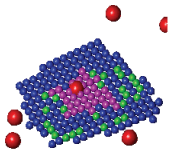

Figure 1: Figure showing various processes involved in the simulation. Plot of the concentration profiles of the various intracellular proteins and the cell-mass over a period of 200 hours for one automaton cell in the model. This is obtained by solving the system of equations, Equations 1 to 6 with the relevant parameter values from Powathil et al. [36] and the plot below shows a representative realisation of the spatial distribution of oxygen $(K)$ or drugs $\left(C_{i}\right)$, obtained by solving the corresponding equations. 


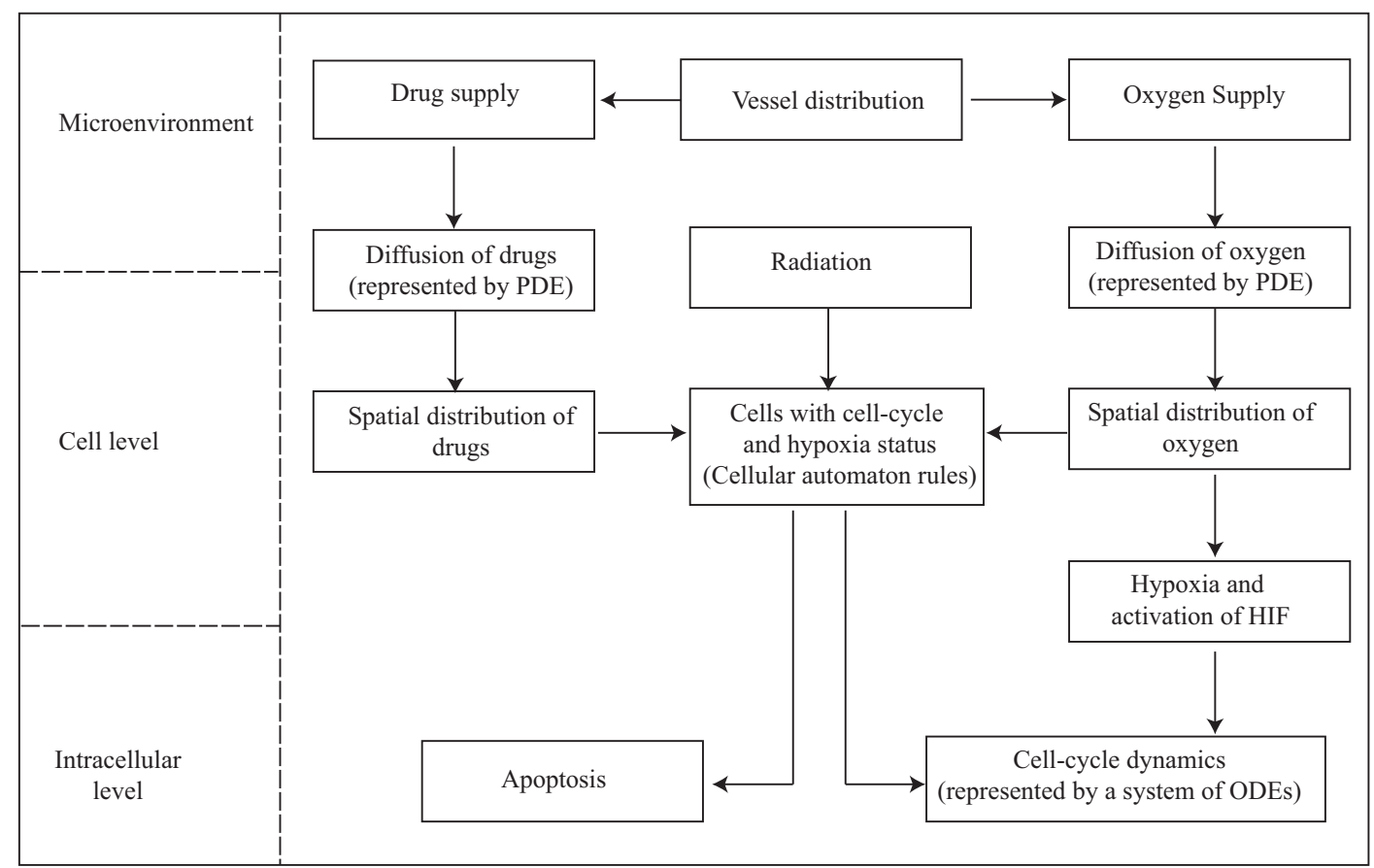

Figure 2: Schematic diagram of the model showing the appropriate scales involved.

(i)
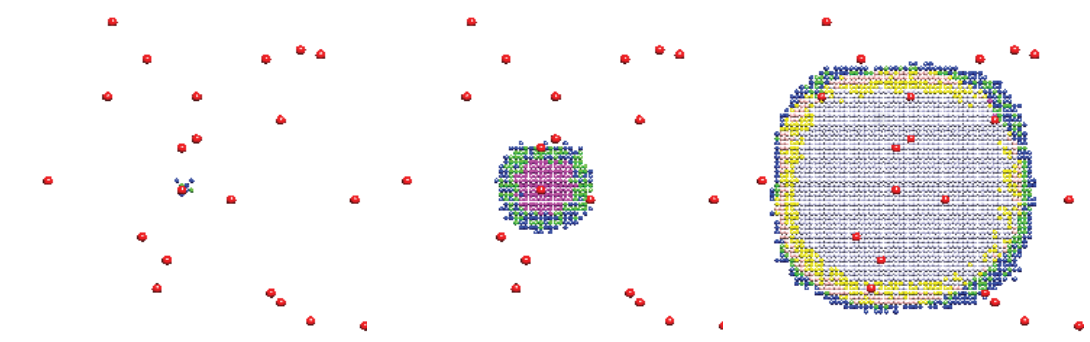

(a) Time $=100 \mathrm{hr}$

(b) Time $=300 \mathrm{hr}$

(c) Time $=600 \mathrm{hr}$

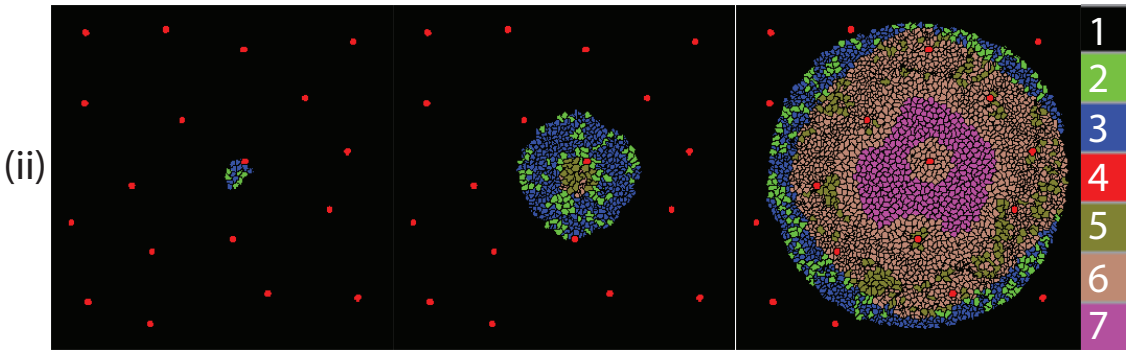
(a) Time $=150 \mathrm{hr}$
(b) Time $=350 \mathrm{hr}$
(c) Time $=650 \mathrm{hr}$

Figure 3: Plots of the spatial distribution of the cells in different stages of the cell cycle. (i) Plots from hybrid multiscale CA model and the colours represent various stages of cell-cycle, namely G1 (blue), S-G2-M (green), resting (magenta), hypoxic cells in G1 (rose), hypoxic cells in S-G2-M (yellow) and hypoxic cells in resting (silver) at times 100, 300 and 600. (ii) Plots from hybrid cellular potts model (using Compucell3D) and the colour legend shows the types of the tumour cells; 1- medium, 2- G2 phase, 3- G1 phase, 4- vessel cross sections, 5- hypoxic G2 phase, 6- hypoxic G1 phase and 7 - resting cells 


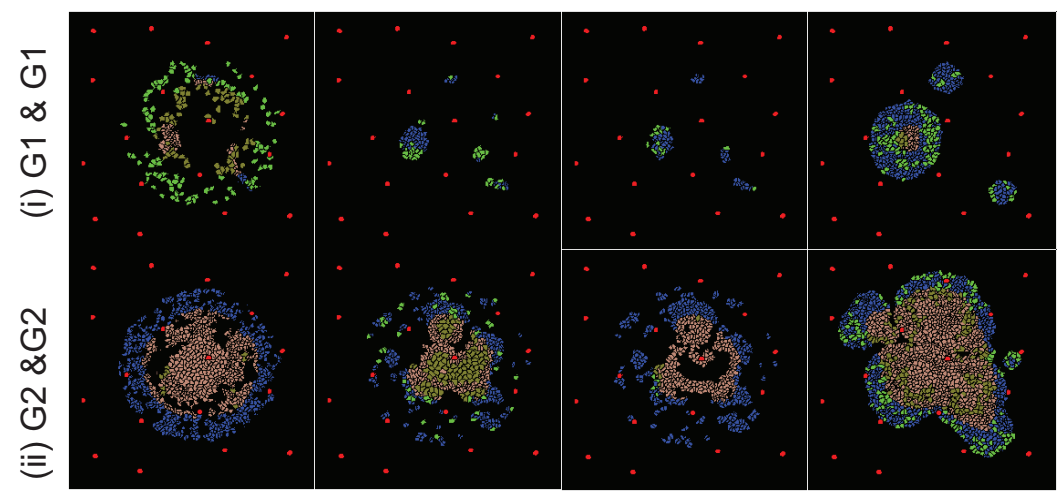

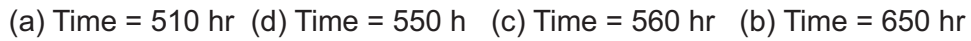

Figure 4: Plots showing the spatial evolution of tumour cells when cell-cycle phase specific chemotherapeutic drugs are given. (i) G2 drug followed by G2 drug and (ii) G1 drug followed by G1 drug at times (a) $510 \mathrm{hr}$, (b) $550 \mathrm{hr}$, (c) 560 hr and (d) 750 hr. Adapted from Powathil et al.[56]

(i)

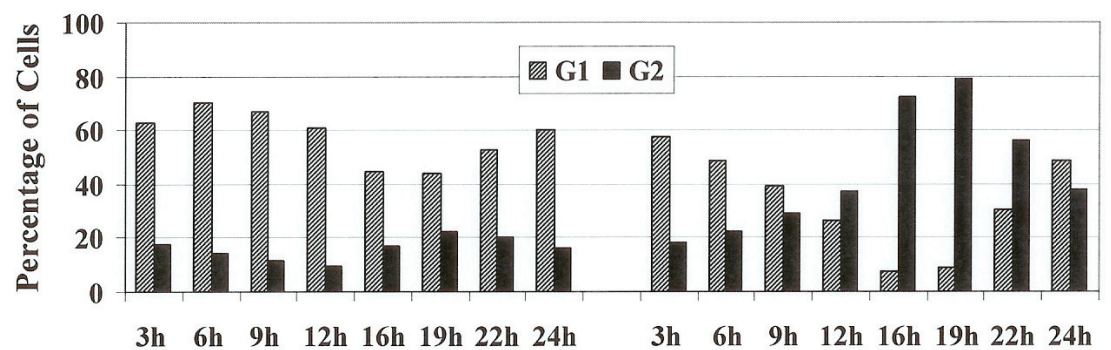

0 Gy $\quad 3$ Gy

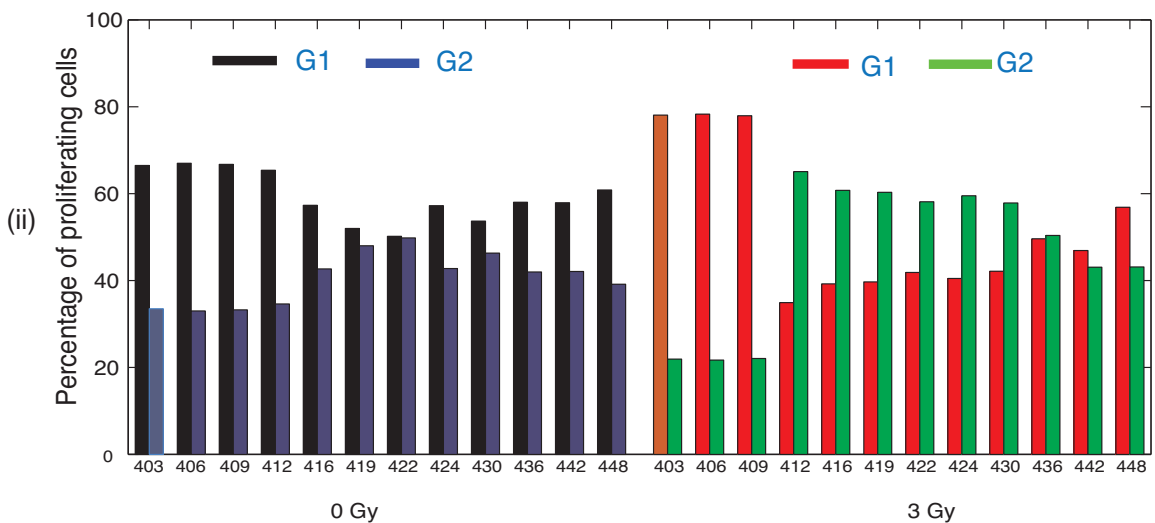

Figure 5: Percentage of cells in G1 and G2 states with and without radiation (a) experimental results from [59]. Cells were irradiated with 3 Gy and samples were taken after $3 \mathrm{~h}, 6 \mathrm{~h}, 9 \mathrm{~h}, 12 \mathrm{~h}, 16 \mathrm{~h}, 22 \mathrm{~h}$, and 24 h for flow cytometry analysis. The cell cycle distribution of irradiated cells was compared to unirradiated cells collected at the same time points. The left peak in each case represents cells in G1 and the right peek represents cells in G2 phase. From Chaudhry [59] (BioMed Central OpenAccess). and (b) simulation results (percentage of proliferating cells). The percentage of proliferating cells in G1 and G2 phases when the cell is irradiated with dose $=3$ Gy at time $=400,403,406,409,412,416$, 419, 422, 424, 430, 436, 442 and $448 \mathrm{~h}$. The cell-cycle distribution of irradiated cells was compared to non-irradiated cells collected at the same time points. The results show a qualitative agreement with the experimental results. Adapted from [52]. 